\title{
Probing Nanostructures Site by Site with the Aberration-Corrected STEM
}

S. J. Pennycook ${ }^{1,2}$, A. R. Lupini ${ }^{1}$, M. Varela ${ }^{1}$, A. Borisevich ${ }^{1}$, Y. Peng ${ }^{1}$, R. Buczko ${ }^{1,2,3}$, X. Fan ${ }^{1,4}$, J. R. McBride ${ }^{5}$, T. C. Kippeny ${ }^{5}$, S. J. Rosenthal ${ }^{5}$, A. Franceschetti ${ }^{1,2}$ and S. T. Pantelides ${ }^{1,2}$

${ }^{1}$ Condensed Matter Sciences Division, Oak Ridge National Laboratory, Oak Ridge, TN

${ }^{2}$ Department of Physics and Astronomy, Vanderbilt University, Nashville, TN

${ }^{3}$ Institute of Physics, Polish Academy of Sciences, 02-668 Warsaw, Poland

${ }^{4}$ Center for Advanced Microscopy, Michigan State University, East Lansing, MI

${ }^{5}$ Department of Chemistry, Vanderbilt University, Nashville, TN

The combination of atomic-resolution Z-contrast microscopy, electron energy loss spectroscopy and first-principles theory has proved to be a powerful means for structure property correlations at interfaces, providing a depth of insight that no technique alone could provide [1]. Similar synergistic advantages are now becoming apparent with nanomaterials, where electron microscopy has the unique advantage of being able to probe individual nanostructures [2]. Indeed, with the success of aberration correction in STEM, it appears feasible to probe individual nanostructures site by site, with single atom sensitivity.

Figure 1 illustrates this synergy for the metal-catalyzed laser ablation growth of single wall carbon nanotubes. From the image it is possible to see the structure and 3D nature of a small catalyst cluster, and EELS profiles indicate a uniform alloy composition. The key issue concerns the growth mechanism, and even with an in-situ microscope it would not be feasible to observe the initial nucleation of solid carbon on the surface of a cooling liquid metal-carbon droplet. Theory however has no such limitations, and Fig. 2 shows calculations of the total energy of a variety of possible carbon nuclei on a Ni surface. Despite the higher energy cost of pentagons compared to hexagons, the pentagons allow the structure to bend, which allows the dangling bonds around the periphery of the flake to saturate in the metal surface. The lowest energy structure on a metal surface is a hemispherical cap or capped tube. Subsequently the structure can elongate by a root growth mechanism.

Figure 2 shows CdSe nanocrystals observed with the VG Microscopes HB603U STEM shortly after installation of a Nion aberration-corrector. With a probe size of $\sim 0.9 \AA$, nanocrystals are resolved in several zone axes. The improved signal to noise ratio and image contrast is advantageous for $3 \mathrm{D}$ reconstruction of nanocrystal shape. A single image is sufficient, provided the thickness dependence of the Z-contrast image is known, and assuming the shape reflects the symmetry of the crystal. However, it is apparent from Fig. 1 that the as-grown nanocrystals do not have well-formed facets. Recently, optical absorption measurements have indicated that etching the nanocrystals in butylamine creates 3 magic sizes with absorbances at $360 \mathrm{~nm}, 390 \mathrm{~nm}$ and $414 \mathrm{~nm}$. These are expected to be well-facetted and amenable to $3 \mathrm{D}$ reconstruction. Again, we anticipate theory to be able to illuminate the nucleation pathway and probe the nature of the stable facets and the electronic consequences of point defects on the surfaces. With the aberration-corrected probe, such defects may even be detectable through EELS. 


\section{References:}

[1] M. Kim et al, Phys. Rev. Lett. 86 (2001) 4056

[2] S. J. Pennycook et al., Proc. MRS 748 (2003) G1.1

[3] This work was supported by the USDOE under contract DE-AC05-00OR22725 managed by UTBattelle, LLC.
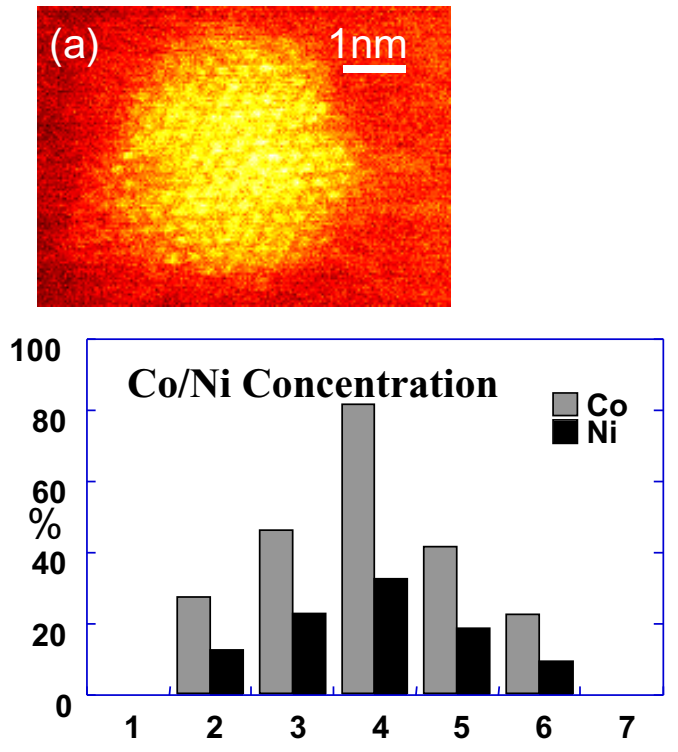

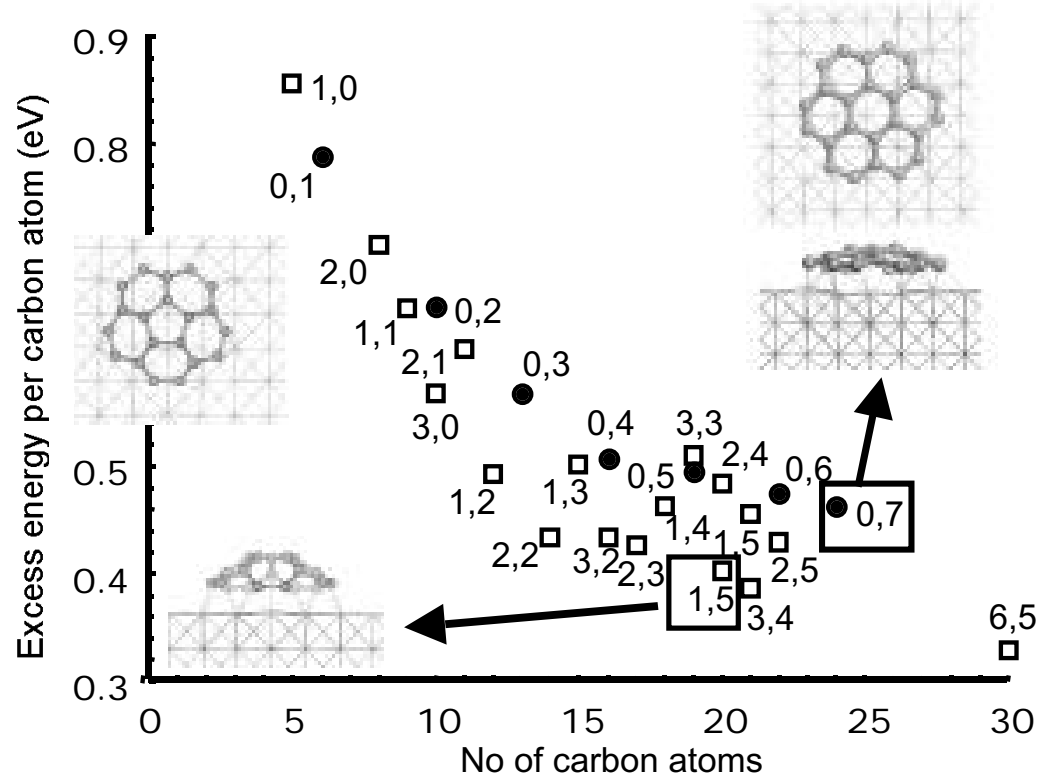

Fig. 1. (a) Z-contrast image of a $4 \mathrm{~nm}$ catalyst nanoparticle used for laser ablation growth of single wall carbon nanotubes. (b) EELS profile across a $2 \mathrm{~nm}$ nanoparticle. (c) Determination of the nucleation pathway from first-principles calculations. Structures containg hexagons are shown as solid points, structures containing pentagons as squares. Points are labeled according to the number of (pentagons, hexagons) in each structure.

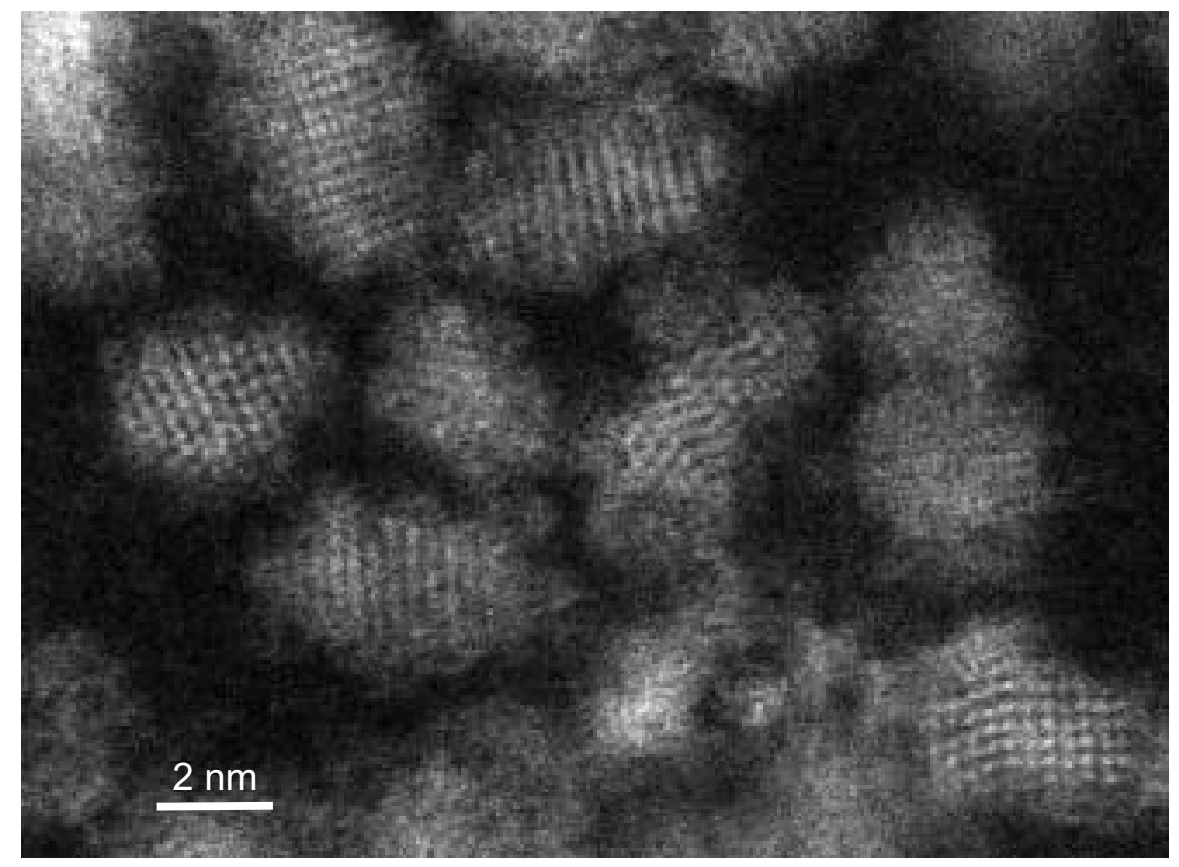

Fig. 2. Images of CdSe

nanocrystals on a carbon support film showing a variety of zone axes. 